\title{
Prolonged surveillance of colorectal cancer patients after curative surgeries beyond five years of follow-up
}

\author{
Lingheng Kong", Jianhong Peng", Jibin Li, Fulong Wang, Cong Li, Peirong Ding, Liren Li, Gong Chen, \\ Xiaojun Wu, Zhenhai Lu, Yujing Fang, Zhizhong Pan, Desen Wan \\ Department of Colorectal Surgery, Sun Yat-sen University Cancer Center, State Key Laboratory of Oncology in South China, Collaborative \\ Innovation Center for Cancer Medicine, Guangzhou 510060, China \\ Contributions: (I) Conception and design: D Wan, L Kong, J Peng; (II) Administrative support: All authors; (III) Provision of study materials or \\ patients: F Wang, C Li, P Ding, L Li, G Chen, X Wu, Z Lu, Z Pan; (IV) Collection and assembly of data: J Peng, J Li, Y Fang; (V) Data analysis and \\ interpretation: J Li, J Peng; (VI) Manuscript writing: All authors; (VII) Final approval of manuscript: All authors. \\ \#These authors contributed equally to this work. \\ Correspondence to: Desen Wan. Department of Colorectal Surgery, Sun Yat-sen University Cancer Center, State Key Laboratory of Oncology in South \\ China, Collaborative Innovation Center for Cancer Medicine, 651\#, Dongfengdong Road, Guangzhou 510060, China. Email: wands@sysucc.org.cn.
}

\begin{abstract}
Background: Local or distant recurrence may develop beyond 5 years after radical resection for colorectal cancer (CRC). There is little evidence of a pattern of recurrence after the routinely recommended 5 years of follow-up. We aimed to investigate the efficacy of the prolonged follow-up beyond 5 years.

Methods: We retrospectively analyzed clinical and survival data of 1,054 CRC patients who underwent radical resections from 1980 to 1996 in our center. The prolonged surveillance was recommended for each patient with a duration of over 20 years.

Results: The follow-up rates of 5, 10, 15, and 20 years were $92.6 \%, 86.9 \%, 82.3 \%$ and $76.8 \%$, respectively. Overall survival (OS) rates of 5, 10, 15, and 20 years were $68.4 \%, 57.7 \%, 52.6 \%$ and 45.0\%, respectively. Totally, $112(10.6 \%)$ patients developed local recurrences and 174 (16.5\%) patients developed distant metastases. The $99.2 \%$ postoperative local recurrences and distant metastases occurred within the first 15 years of surveillance. Survival differed between four age groups. Local recurrence was mainly diagnosed among rectal cancer patients, especially in those with lower-third rectal cancer. Metastases were commonly found in the liver and lungs. Patients with colon cancer and stage I/II manifested significantly longer OS than patients with rectal cancer and stage III/IV (both $\mathrm{P}<0.001$ ).

Conclusions: In this study, postoperative local recurrences and distant metastases was rarely found after 15 years of enhanced surveillance, which indicated a "true cure" if the patient did not develop recurrences and metastases after 15 years.
\end{abstract}

Keywords: Colorectal cancer (CRC); radical resection; long-term follow-up

Submitted Sep 28, 2019. Accepted for publication Oct 08, 2019.

doi: 10.21037/atm.2019.10.39

View this article at: http://dx.doi.org/10.21037/atm.2019.10.39

\section{Introduction}

Nearly $50 \%$ of colorectal cancer (CRC) patients suffer from local recurrence or metastasis even if radical operations are performed. Intense follow-up is encouraged during the first 5 years after surgery, which aimed at early detection of relapse. Therefore, current guidelines recommend surveillance every 3 to 6 months for 2 years, then every
6 months for a total of 5 years (1).

However, a small but nonnegligible portion of treatment failure occurs beyond regular surveillance duration, and thus a "true cure" cannot be confidently ensured at the end of the 5 -year of follow-up. Moreover, resection of metachronous colorectal liver metastasis results in a promising prognosis. A few patients with late onset of relapse may benefit from 
prolonged surveillance.

Thus far, there is little evidence showing a pattern of late relapse after CRC resection and supporting an ideal length of follow-up. In this study, we aimed to investigate the efficacy and possible endpoint of enhanced surveillance and the relapse pattern of CRC patients who received radical resection by a prolonged follow-up for more than 20 years.

\section{Methods}

\section{Patients}

The clinical records of consecutive patients who underwent radical resection for CRC between January 1980 and December 1996 at Sun Yat-sen University Cancer Center were retrospectively reviewed. All patients were histologically confirmed colorectal adenocarcinoma without preoperative distant metastasis. Patients were excluded when radical resection could not be performed or surveillance information could not be obtained. The general patient characteristics, tumor traits, and follow-up data were recorded from our both electronic medical record system and follow-up tracking system. A right-sided colon was defined as a bowl between the appendix and transverse colon; a left-sided colon was defined as bowl between the splenic flexure and the sigmoid colon. The rectal cancer was divided into three parts according to its location with less than $5 \mathrm{~cm}, 5$ to $10 \mathrm{~cm}$, and more than $10 \mathrm{~cm}$ from the anal verge. CRC staging was classified according to the 2017 American Joint Committee on Cancer staging system. The study was approved by the ethics committee at Sun Yat-sen University Cancer Center. Written informed consent was obtained from all patients (approval number: B2017-042-01).

\section{Follow-up}

All patients were followed up via outpatient clinic, mail, and telephone interview. The post-operation follow-up interval was 3 months in the first 2 years and then 6 months in the following 3 years. Annual follow-up was recommended after 5 years of survival. Physical examination, ultrasound, X-ray, and computed tomography (CT) scan were used to detect local recurrence and distant metastasis. The follow-up was closed in June 2017.

\section{Statistical analysis}

Categorical data were presented as number (percentage) and further compared by using chi-square test. OS rates were calculated by Kaplan-Meier method. Log-rank test was used to compare differences of survival rates between groups. A two-sided $\mathrm{P}$ value of $<0.05$ was considered statistically significant. All statistical analyses were performed using SPSS statistical software (version 22.0, Chicago, IL, USA).

\section{Results}

\section{Patient characteristics}

A total of 1,054 CRC patients were included with a median age of 54 years (range, 20-84 years). Among whom, 598 patients $(56.7 \%)$ were male. There were $159(15.1 \%)$, $186(17.6 \%)$ and $653(62.0 \%)$ tumors located in the rightsided colon, left-sided colon, and rectum, respectively. In total, 142 cases (13.5\%) were classified as stage I, 587 cases (55.7\%) were stage II, and 325 cases (30.8\%) were stage III (Table 1).

\section{Survival outcome}

The median follow-up time was 8.7 years (range, 3-20 years). The follow-up rates of 5, 10, 15, and 20 years were $92.6 \%, 86.9 \%, 82.3 \%$, and $76.8 \%$, respectively. Cumulative OS rates of 5, 10, 15 and 20 years were $68.4 \%$, $57.7 \%, 52.6 \%$, and 45.0\%, respectively (Table 2, Figure 1).

Survival analysis showed that the prognosis was related to age $(\mathrm{P}=0.026$, Figure 2$)$, site $(\mathrm{P}<0.001$, Figure 3$)$, and stage $(\mathrm{P}<0.001$, Figure 4). No significant relationship between gender, differentiation, and survival was found.

Subgroup analysis showed that compared with those who died within 5 years due to CRC, patients who survived over 15 years had a higher proportion of colon cancer and stage I and II disease (Table 3).

\section{Postoperative relapse outcome}

In total, $112(10.6 \%)$ patients developed local recurrences and $174(16.5 \%)$ patients developed distant metastases. Among them, 22 patients suffered from both local recurrences and distant metastases. Among the patients developing postoperative relapse, $91.3 \%$ relapse was found in the first 5 years of follow-up, which is highest proportion of the period. Then, the proportion of relapse decreased as surveillance was prolonged. After 15 years of surveillance, there were only $0.8 \%$ patients were found relapse (Table 4 ).

As shown in Table 5, the local recurrence which mainly 
Table 1 Clinicopathological characteristics of colorectal cancer patients after radical resection

\begin{tabular}{|c|c|c|c|}
\hline Characteristics & Subgroup & Patients $(n=1,054)$ & Percentage (\%) \\
\hline Gender & Female & 456 & 43.3 \\
\hline \multirow[t]{3}{*}{ Age (years) } & $<30$ & 37 & 3.5 \\
\hline & $30-49$ & 341 & 32.4 \\
\hline & $\geq 70$ & 85 & 8.1 \\
\hline \multirow[t]{4}{*}{ Tumor site } & Right-sided colon & 159 & 15.1 \\
\hline & Left-sided colon & 186 & 17.6 \\
\hline & Upper rectum & 34 & 3.2 \\
\hline & Multiple lesions & 29 & 2.8 \\
\hline \multirow[t]{6}{*}{ Surgery procedure } & Right hemicolectomy & 143 & 13.6 \\
\hline & Left hemicolectomy & 80 & 7.6 \\
\hline & Sigmoidectomy & 98 & 9.3 \\
\hline & Transverse colon resection & 19 & 1.8 \\
\hline & Anterior resection & 211 & 20.0 \\
\hline & Abdominal perineal resection & 490 & 46.5 \\
\hline \multirow{4}{*}{ Differentiation } & Moderately & 441 & 41.8 \\
\hline & Poor & 93 & 8.8 \\
\hline & Undifferentiated & 6 & 0.6 \\
\hline & Undefined & 374 & 35.5 \\
\hline \multirow[t]{3}{*}{ Stage } & I & 142 & 13.5 \\
\hline & II & 587 & 55.7 \\
\hline & III & 325 & 30.8 \\
\hline
\end{tabular}

originated from rectal cancers, especially those located in the lower third of the rectum and anus. Distant metastases were detected mostly in the liver (39.1\%), lungs (36.2\%), and peritoneum (20.7\%). However, there was no obvious relation between tumor site and distant metastatic rate.

\section{Discussion}

$\mathrm{CRC}$ is one of the most curable cancers of the digestive tract. However, according to global cancer statistics, incidence and mortality of CRC are still rising. It was estimated that in 2018 there were 1,804,000 new CRC 
Table 2 Cumulative survival rate of colorectal cancer patients after radical resection

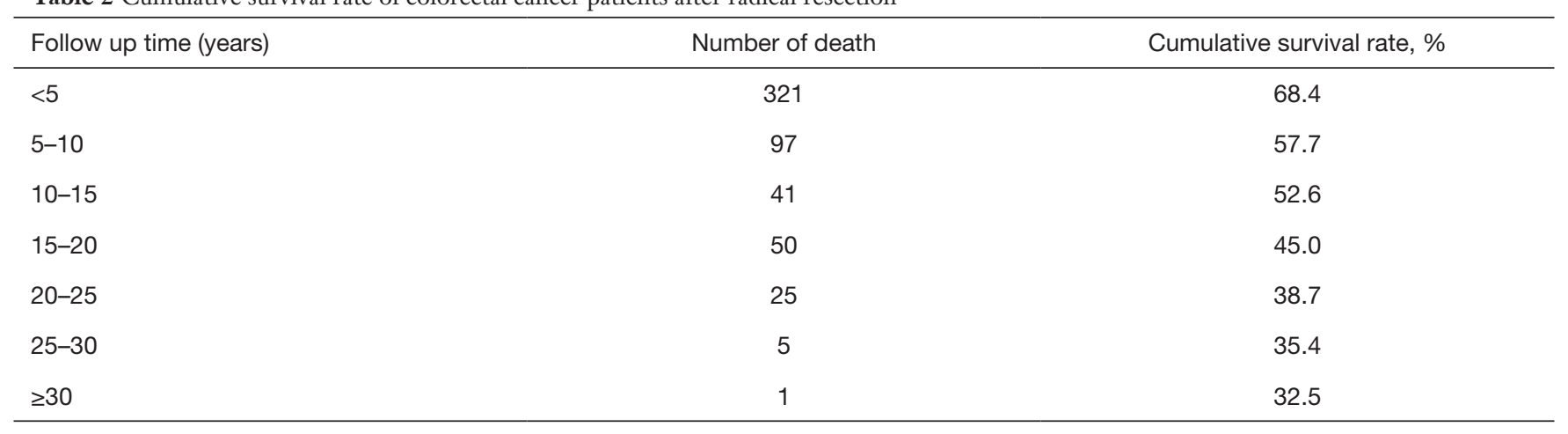

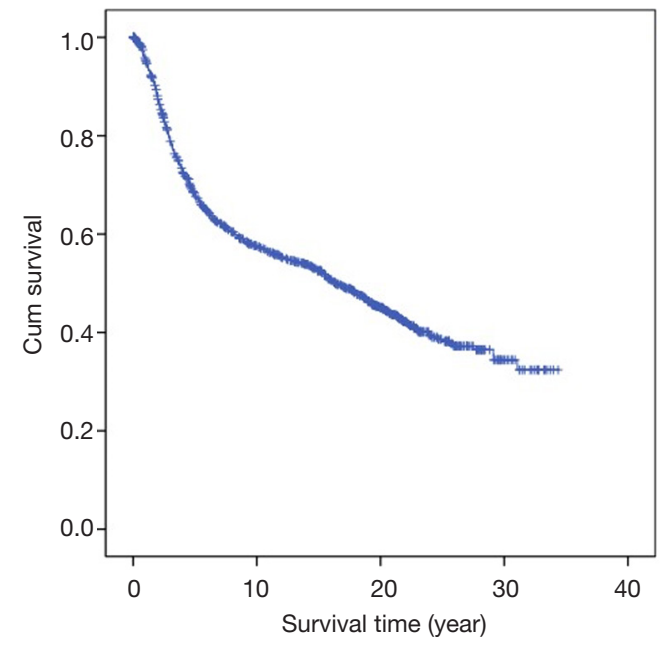

Figure 1 Kaplan-Meier curve of cumulative overall survival.

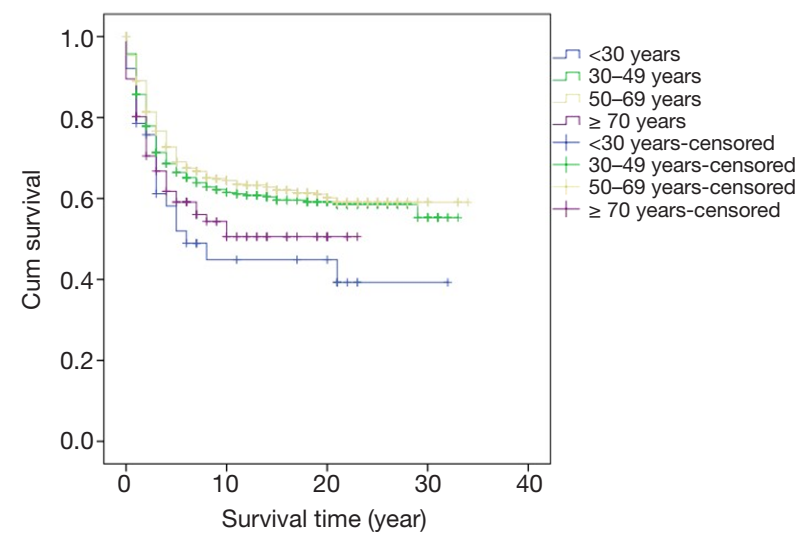

Figure 2 Kaplan-Meier curve of overall survival by comparison of age.

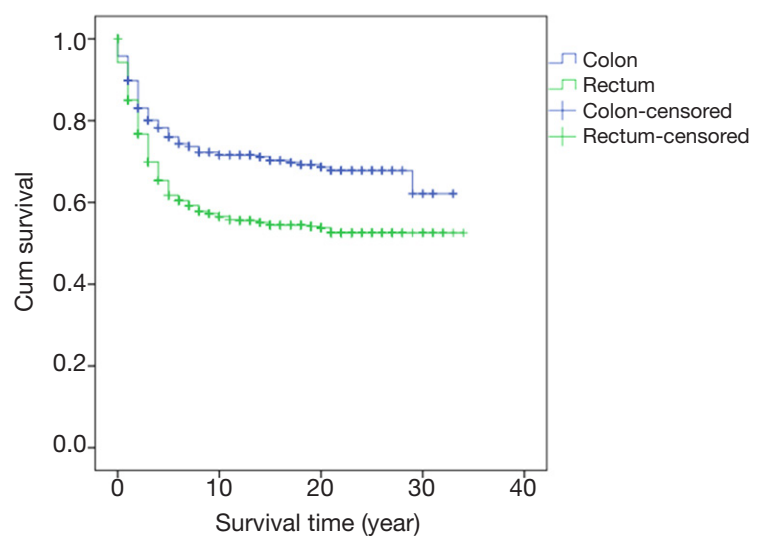

Figure 3 Kaplan-Meier curve of overall survival by comparison of tumor site.

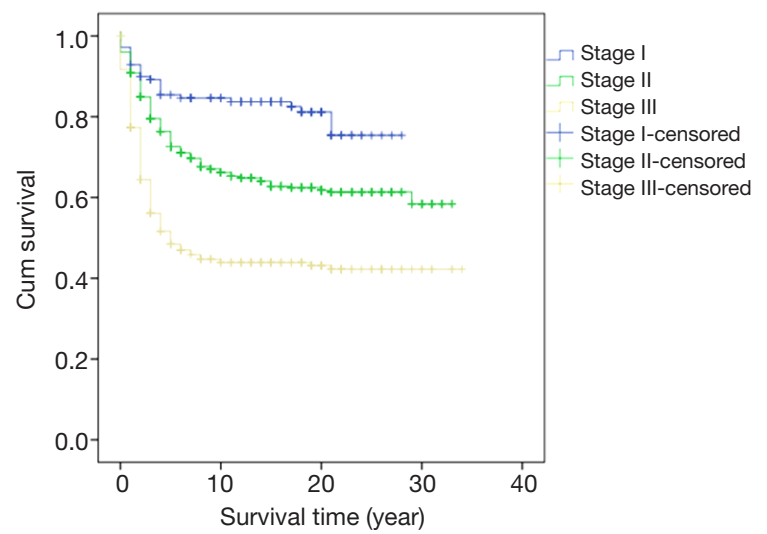

Figure 4 Kaplan-Meier curve of overall survival by comparison of stage. 
Table 3 Differences of clinicopathological characteristics between patients surviving less than 5 years and over 15 years

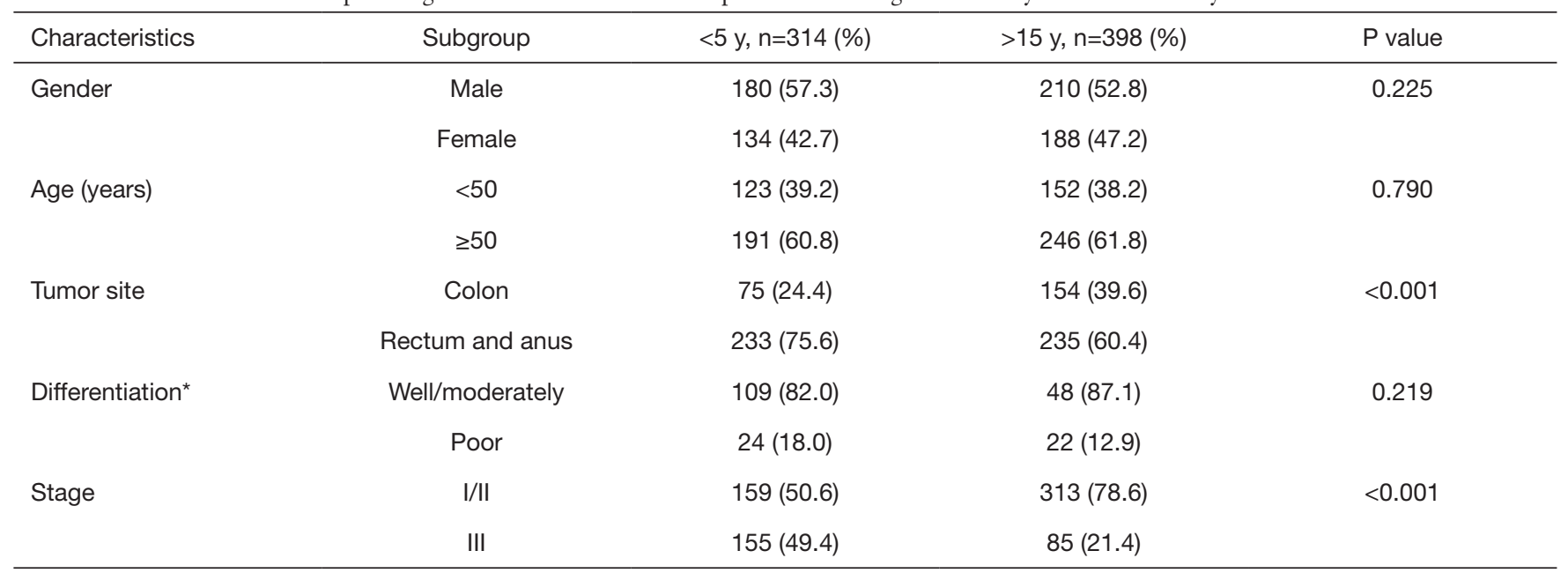

*, 29 cases with undefined differentiation were excluded.

Table 4 Recurrence and metastasis by follow up time

\begin{tabular}{lcc}
\hline Follow-up time (years) & Number of recurrence/metastasis & Percentage (\%) \\
\hline$<5$ & 241 & 91.3 \\
$5-10$ & 16 & 6.1 \\
$10-15$ & 5 & 1.9 \\
$15-20$ & 2 & 0.8 \\
$20-25$ & 0 & 0 \\
$25-30$ & 0 & 0 \\
$\geq 30$ & 0 & 0 \\
\hline
\end{tabular}

Table 5 Local recurrence and metastasis rate according to tumor site

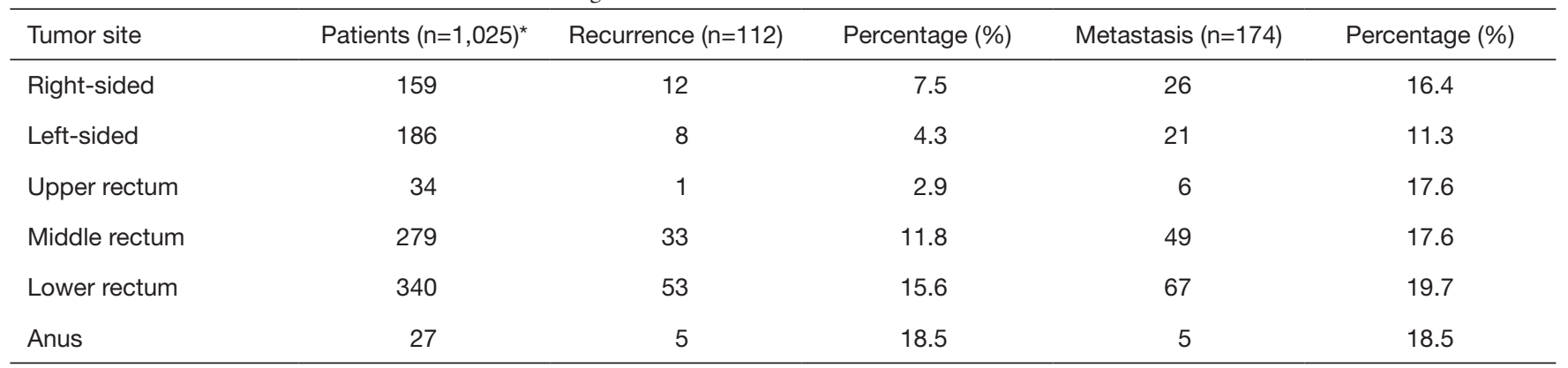

*, 29 cases with multiple lesions were excluded.

cases worldwide causing 862,000 deaths, which increased by $32.5 \%$ and $24.2 \%$ in comparison to those in 2012 , respectively $(2,3)$. In China, CRC is the third most common malignancy and accounts for 187,000 deaths annually (4).
Surgery is still the principal treatment for CRC without metastasis. Relapse occurs in $30-50 \%$ cases after curative surgery and mainly involves the liver, lung and surrounding tissues (5). Survival rates vary from more than $39 \%$ to $70 \%$ 
in different countries (6). Recent studies have demonstrated that active regimens for resectable metastatic lesions may result in an encouraging prognosis. Surveillance aiming at early detection of metastasis in the first few years after surgery has been approved by most clinicians.

Few studies have provided evidence on the optimal duration of enhanced follow-up. Frequent examinations undoubtedly impair the social function and psychological health of the majority of cured patients. The benefit of the survival and cost-effectiveness of frequent follow-up has been doubted (7). Renehan et al. pooled data from 5 clinical trials comprising 1,342 patients, and concluded that there were no differences between recurrence rates of the frequent surveillance and control group, apart from an 8.5-month-earlier time for the detection of relapse (8). Another meta-analysis by Bruinvels et al. analyzed 7 nonrandomized trials and demonstrated that frequent follow-up may result in finding more asymptomatic relapse and more cases suitable for further operation (9).

In this study, we identified the pattern of relapse by prolonging follow-up to 20 years, and discovered that a quarter of curatively resected CRC cases will develop recurrence or metastasis, $91.3 \%$ of which occur within the first 5 years. During the following years of surveillance, the possibility of finding relapse decreases gradually to $0.8 \%$ beyond 15 years. We can confidently declare "true cure" in a patient by following up so extensively. Patients can return to normal life without extra psychological and economic burden.

In this study, the five-year cumulative overall survival (OS) rate was $68.4 \%$, which is similar to most related studies (6). Factors related to survival include age, stage, and tumor site. We found that patients younger than 30 years and older than 70 years may have worse prognosis. Chiarg et al. also concluded that age is a prognostic factor (10). Again, stage turns out to be another strong predictive parameter of prognosis. Like many other studies, we demonstrated that an early stage of disease is significantly related to lower mortality $(11,12)$.

The significance of the tumor site has also been a major topic of discussion recently. Most treatment failures of rectal cancer are mainly due to local recurrence. A possible reason for this result was the absence of perioperative regimens three decades ago. An update of the prognosis of rectal cancer with the latest regimen needs to be investigated. The right-sided and left-sided colon present different survival possibilities as well. Benedix et al. reported that the left-sided colon demonstrated better OS and disease-free survival (13).

The liver is the commonest organ of CRC metastasis. It has been reported that CRC liver metastasis occurs in $15-25 \%$ cases at the time of diagnosis and in $25-50 \%$ 3 years after primary treatment (14). Fortunately, resectable liver disease results in a promising five-year survival rate of $25-58 \%(14,15)$. Similarly, the survival rate of resectable lung metastasis has reached $21-26 \%(16,17)$. Thus, the known pattern of relapse enables exact enhanced surveillance in order to discover as many cases of curable relapse as possible.

\section{Conclusions}

In summary, postoperative recurrence is rarely seen 15 years after radical CRC surgery, implying that surveillance can be ended beyond this point, but enhanced surveillance is strongly recommended in the early years.

\section{Acknowledgments}

We deeply appreciate the help we received from all colleagues of the Department of Colorectal Surgery at Sun Yat-sen University Cancer Center who were involved in performing the treatments used in the current study.

Funding: This work was supported by the Guangzhou Science and Technology Plan Projects (Health Medical Collaborative Innovation Program of Guangzhou) (grant No. 201803040019), the National Natural Science Foundation of China (grant No. 81871991 and No. 81772595), and Science and Technology Planning Project of Guangdong Province (grant No. 2013B090800047).

\section{Footnote}

Conflicts of Interest: The authors have no conflicts of interest to declare.

Ethical Statement: The authors are accountable for all aspects of the work in ensuring that questions related to the accuracy or integrity of any part of the work are appropriately investigated and resolved. The study was approved by the ethics committee at Sun Yat-sen University Cancer Center. Written informed consent was obtained from all patients (approval number: B2017-042-01).

Disclaimer: The authenticity of this article has been validated by uploading the key raw data onto the Research Data Deposit public platform (http://www.researchdata.org.cn), with the Approval Number as RDDA2019001154. 


\section{References}

1. Rex DK, Kahi CJ, Levin B, et al. Guidelines for colonoscopy surveillance after cancer resection: a consensus update by the American Cancer Society and the US Multi-Society Task Force on Colorectal Cancer. Gastroenterology 2006;130:1865-71.

2. Bray F, Ferlay J, Soerjonmataram I, et al. Global Cancer Statistics 2018; GLOBOCAN estimates of incidence and mortality worldwide for 36 cancers in 185 countries.CA cancer J Clin 2018;68:394-424.

3. Steward BW, Wild CP. World Cancer Report. IARC WHO Iyon 2014.

4. Zheng RS, Sun K, Zhang S, et al. Report of cancer epidemiology in China, 2015. Zhonghua Zhong Liu Za Zhi 2019;41:19-28.

5. Tjandra JJ, Chan MKY. Follow-up after curative resection of colorectal cancer: A meta-analysis Dis Colon Rectum 2007;50:1783-99.

6. Allemani C, Matsuda T, Carlo VD, et al. Global Surveillance of trends in cancer: analysis of individual records for 37513025 patients diagnosed with one of 18 cancers during 2000-2014 from 322 populationbased registries in 71 countries (CONCORD-3). Lancet 2018;391:1023-75

7. Virgo KS, Wade TP, Longo WE, et al. Surveillance after curative colon cancer resection: practice patterns of surgical subspecialists. Ann Surg Oncol 1995;2:472-82.

8. Renehan AG, Egger M, Saunders MP, et al. Impact on survival of intensive follow up after curative resection for colorectal cancer: systematic review and meta-analysis of randomised trials. BMJ 2002;324:813-6.

Cite this article as: Kong L, Peng J, Li J, Wang F, Li C, Ding P, Li L, Chen G, Wu X, Lu Z, Fang Y, Pan Z, Wan D. Prolonged surveillance of colorectal cancer patients after curative surgeries beyond five years of follow-up. Ann Transl Med 2019;7(21):608. doi: 10.21037/atm.2019.10.39
9. Bruinvels DJ, Stiggelbout AM, Kievit J, et al. Follow-up of patients with colorectal cancer: A meta-analysis. Ann Surg 1994;219:174-82.

10. Chiang JM, Chen MC, Changch CR, et al. FFavorable influence of age on tumor characteristics of sporadic colorectal adenocarcinoma: patients 30 years of age or younger may be a distinct patient group. Dis Colon Rectum 2003;46:904-10.

11. Compton CC, Fielding LP, Burgart LJ, et al. Prognostic factor in colorectal cancer: college of American pathologist consensus statement. Arch Pathol Lab Med 2000;124:979-94.

12. Walker J, Quirke P, Prognosis and response to therapy in colorectal cancer. Eur J Cancer 2002;38:880-6.

13. Benedix F, Kube R, Meyer F, et al. Comparison of 17641 patients with right-and left-sided colon cancer: differences in epidemiology, perioperative course, histology, and survival. Dis Colon Rectum 2010;53:57-64.

14. Lupinacci R, Penna C, Nordlinger B. Hepatectomy for resectable colorectal cancer metastases-indicators of prognosis, definition of resectability, techniques and outcomes. Surg Oncol Clin N Am 2007;16:493-506.

15. Kuvshinoff B and Fong Y. Surgical therapy of liver metastases. Seminars in Oncology 2007;34:177-85.

16. Ogata Y, Matono K, Hayashi A, et al. Repeat pulmonary resection for isolated recurrent lung metastases yields result comparable to those after first pulmonary resection in colorectal cancer. World J Surg 2005;29:363-8.

17. Ike H, Shimadu H, Ohki S, et al. Results of aggressive resection of lung metastases from carcinoma detected by intensive follow-up. Dis Colon Rectum 2002;45:468-73. 